\title{
Therapeutic approaches to severe and cerebral malaria and design of trials
} Arjen M Dondorp

Address: Mahidol-Oxford Research Unit, Faculty of Tropical Medicine, Mahidol University, Bangkok 10400, Thailand Email: Arjen M Dondorp - arjen@tropmedres.ac

from Infectious diseases of the nervous system: pathogenesis and worldwide impact Paris, France. 10-13 September 2008

Published: 23 September 2008

BMC Proceedings 2008, 2(Suppl I):S9

This abstract is available from: http://www.biomedcentral.com/I753-656I/2/SI/S9

(c) 2008 Dondorp; licensee BioMed Central Ltd.

Severe and cerebral malaria is a global problem, claiming at least 1 million lives annually. Case fatality rates are high; around $10 \%$ in African children and $20 \%$ in adults in SE Asia, but mortality is higher when multiple organs are affected. Management of patients with severe malaria can be divided into:

1. Antimalarial treatment; 2 . Treatment of concomitant diseases; 3 . Supportive treatment; and 4. Adjunctive treatments.

Prompt start of parenteral antimalarial is essential. The antimalarial treatment of severe disease is being transformed by the development and deployment of the watersoluble artemisinin derivative artesunate. Parenteral artesunate is now the treatment of choice in low-transmission areas and in the 2nd and 3rd trimesters of pregnancy, and research is underway into whether it should replace quinine as the treatment of choice in African children. The development of artesunate suppositories may provide the means to treat patients with severe disease in remote rural settings, potentially buying the time needed to reach a health care facility.

In areas of high transmission (sub-Saharan Africa), a significant number of patients (up to $17 \%$ ) with severe febrile illness, including coma, in the presence of a peripheral blood slide with asexual forms of $P$. falciparum have concomitant bacteraemia. A high background prevalence of peripheral blood parasitaemia, as well as the predisposition of severe malaria for bacteraemia contributes to this. A low threshold for administration of broad spectrum antibiotics is warranted in this setting.

Adequate supportive care of complications, preferentially in a high dependency or intensive care setting, is essential to further safe lives. This includes prompt treatment of hypoglycaemia and convulsions. Haemofiltration has proven to be superior to peritoneal dialysis for treatment of acute renal failure and invasive mechanical ventilation is needed in case of ARDS, both feared complications of adult severe malaria. Trials on optimal fluid management and the timing of enteral feeding in cerebral malaria are under way.

A large variety of adjunctive treatments based upon specific aspects severe malaria pathophysiology have been tried, but none has proven to lower mortality, whereas some were shown to be harmful. Many of the treatment studies in severe malaria have been underpowered, which should be avoided in the design of future trials. However, only a limited number of interventions can be tested in large multicenter multinational trials. This will necessitate close collaboration between groups, as well as agreement on the research agenda. 